\title{
Surgical Treatment of Carpal Tunnel Syndrome and Lymphedema in Advanced-Stage Upper Extremity Lymphedema
}

\section{Soo-Byn Kim}

Korea University Guro Hospital

Kyung-Chul Moon ( $\square$ boyhaha2000@daum.net)

Korea University Guro Hospital https://orcid.org/0000-0001-6072-261X

\section{Case report}

Keywords: Breast cancer, Carpal tunnel syndrome, Case report, Lymphedema, Lymphovenous anastomosis, Surgery

Posted Date: March 6th, 2021

DOI: https://doi.org/10.21203/rs.3.rs-278621/v1

License: (c) (i) This work is licensed under a Creative Commons Attribution 4.0 International License. Read Full License 


\section{Abstract}

\section{Background}

Recent advances in supermicrosurgery have evolved to treat lymphedema surgically. For patients with carpal tunnel syndrome (CTS) and advanced-stage lymphedema, lymphovenous anastomosis (LVA) may effectively improve lymphedema after carpal tunnel release in patients with CTS and advanced stage lymphedema. However, no studies have reported simultaneous carpal tunnel release and LVA surgeries for patients with CTS and advanced-stage lymphedema.

\section{Case Presentation}

A 60-year-old female with carpal tunnel syndrome and International Society of Lymphology late stage 2 right upper extremity lymphedema following right mastectomy and axillary lymph node dissection and adjuvant chemoradiotherapy for treating breast cancer was admitted to our lymphedema clinic. She developed carpal tunnel syndrome four years after breast cancer surgery. She underwent release of the transverse carpal ligament, followed by four lymphovenous anastomoses at the wrist, forearm, and antecubital area. After two simultaneous surgeries, she had less neuropathic pain and volume reduction in her hand.

\section{Conclusion}

The authors recommend simultaneous LVA and release of the transverse carpal ligament as the first treatment option for patients with advanced-stage lymphedema and concurrent CTS.

\section{Background}

Breast cancer-related lymphedema is a chronic condition characterized by lymphatic vessel ectasia and valve dysfunction, followed by the reflux of lymphatic fluid into the interstitial space. Lymph stasis may lead to a chronic inflammatory process, resulting in adipose tissue differentiation and fibroadipose tissue deposition [1]. Lymphedema is traditionally treated with decongestive physiotherapy and compression garments. Controversy exists regarding a cause-effect association between lymphedema and the development of peripheral neuropathies [2]. However, increasing abnormal fibroadipose tissue deposition and pressure in upper extremity lymphedema patients and repetitive compression garment therapy may act as risk factors for the development of carpal tunnel syndrome (CTS), particularly in patients with advanced-stage lymphedema [3, 4].

Gunnoo et al. [5] demonstrated a significant increase in 5-month postoperative lymphedema volume compared to the preoperative volume in patients with lymphedema and CTS. Recent advances in supermicrosurgery have evolved to treat lymphedema surgically. Koshima et al. made a key modification by introducing supermicrosurgical lymphovenous anastomosis (LVA), which uses lymphatics and 
venules smaller than $0.8 \mathrm{~mm}$ [6]. Therefore, LVA may effectively improve lymphedema after carpal tunnel release in patients with CTS and advanced stage lymphedema.

However, no studies have reported simultaneous carpal tunnel release and LVA surgeries for patients with CTS and advanced stage lymphedema. This case report describes the successful treatment of lymphedema with LVA in a patient with CTS and advanced-stage lymphedema.

\section{Case Presentation}

A 60-year-old female patient was referred to our lymphedema clinic with a 12-year history of chronic, acquired, right lower extremity lymphedema. The patient had a history of right breast cancer, which was treated with right mastectomy and axillary lymph node dissection and chemoradiation therapy. The patient had right upper extremity lymphedema (International Society of Lymphology late stage 2), and first noticed signs and symptoms of numbness and tingling sensation in her right hand affecting the thumb, index, and middle fingers since the symptoms of lymphedema worsened.

The patient underwent electromyography and the nerve conduction study showed moderate CTS in both hands and that the right hand with lymphedema was worse than the unaffected hand. The results of the median sensory nerve conduction test from the middle finger to the wrist showed latencies of 4.6/5.6 and $4.9 / 5.9 \mathrm{~ms}$, respectively. The bilateral median motor and sensory responses showed prolonged latencies and low amplitudes. In addition, the needle electromyography showed polyphasic motor unit action potentials with reduced recruitment patterns in the bilateral abductor pollicis brevis muscles. The patient also complained of severe right upper extremity heaviness, pain, infection, and intermittent sepsis that required hospitalization. She underwent medical treatments including decongestive physiotherapy for years, daily manual and mechanical lymphatic drainage, and wore compression garments. However, the lymphedema symptoms waxed and waned and were refractory to nonsurgical management.

\section{Preoperative Work-Up}

A milliliter of indocyanine green (ICG) mixed with $2 \%$ lidocaine was injected subcutaneously at the second web space of the affected extremity at the bedside. After two hours, fluorescence images of lymphatic vessels were obtained with a near-infrared camera (Moment K; IANC\&S, Seoul, Korea) and showed confluent dermal backflow in the forearm and few patent lymphatic vessels were visualized on the right upper extremity with severe lymphedema. However, magnetic resonance lymphangiography identified functioning vessels in the wrist and forearm. Therefore, we planned simultaneous carpal tunnel release and LVA to treat the severe lymphedema that the patients experienced for more than ten years. Lymphoscintigraphy and magnetic resonance lymphangiography were also performed before surgery (Fig. 1).

The circumference of the affected and unaffected upper extremity was measured at baseline, follow-up, and the last visit using a standardized tape. The circumference of the affected and unaffected extremities was measured in six places, $15 \mathrm{~cm}$ above the elbow, $10 \mathrm{~cm}$ above the elbow, at the elbow 
(using the antecubital crease as a reference point), $10 \mathrm{~cm}$ below the elbow, $15 \mathrm{~cm}$ below the elbow, and at the wrist. The circumference difference ratio was calculated according to the formula: (circumference of affected extremity - circumference of unaffected extremity) / circumference of unaffected extremity $x$ 100. In addition, the volume of the extremity was calculated based on the circumference measures. The volume segment was measured according to the formula of a truncated cone: $V=\pi \times h \times\left(R^{2}+r^{2}+R r\right) / 3$, where $\pi$ is a constant, $h$ is the height, $R$ is the radius on base, and $r$ is the radius on top $[7,8]$. The circumferences of extremities measured at $10 \mathrm{~cm}$ above the elbow and $10 \mathrm{~cm}$ below the elbow was used to calculate the volume of the segment. The volume of the unaffected extremity was also measured and the volume difference ratio was ultimately calculated according to the formula: (volume of affected extremity - volume of unaffected extremity) / volume of unaffected contralateral extremity x 100 [9].

\section{Surgical Technique}

Release of the transverse carpal ligament (TCL) was performed first, followed by LVA. Under general anesthesia, the patient was placed in the supine position and a tourniquet was used. Incisions were made along the palmar crease line. After making an incision in the TCL, the median nerve was identified, dissected, and the TCL was completely released. Subsequently, four lymphatic vessels (two at the wrist, one at the medial forearm, and one at the antecubital fossa) distal to the areas of dermal backflow were marked for microsurgical anastomosis to adjacent small veins for LVA. Intraoperatively, 3-cm-long longitudinal incisions were made under a surgical microscope were made according to the magnetic resonance lymphangiography. After a superficial fascia incision, functioning lymphatics were identified deep in the superficial fascia and one to two functional lymphatic vessels were anastomosed to the adjacent veins using $11-0$ nylon sutures (Fig. 2). Functional drainage was confirmed by the washout of venous blood in the anastomosed vein.

\section{Postoperative Care}

Antibiotics were administered to prevent cellulitis and infection postoperatively. The right arm was compressed and elevated immediately postoperatively and postoperative compression bandage therapy with 35 to $40 \mathrm{mmHg}$ pressure was instituted following surgery.

\section{Clinical Outcomes}

The circumference difference ratios before surgery were $27,26,25,22,18$, and $17 \% 15 \mathrm{~cm}$ above the elbow, $10 \mathrm{~cm}$ above the elbow, at the elbow (antecubital crease), $10 \mathrm{~cm}$ below the elbow, $15 \mathrm{~cm}$ below the elbow, and at the wrist. The ratios was decreased to $20,17,15,13,12$, and $8 \%$ at these six levels, respectively. The volume difference ratio was also decreased from 51 to $31 \%$. The patient had significant circumference and volume reduction of the right hand (Fig. 3). The CTS and lymphedema symptoms have decreased following synchronous TCL release and LVA surgeries.

\section{Discussion}


Breast cancer-related lymphedema is a chronic disease that affects 15 to $30 \%$ of breast cancer survivors [10]. Previous studies related to the presence of neuropathic pain in patients with breast cancer-related lymphedema reported neuropathic pain in $30 \%$ of the patients [11]. In addition, the degree of neuropathic pain in patients with breast cancer-related lymphedema was more often moderate than mild [12]. In this study, symptoms related to CTS developed since lymphedema worsened four years after mastectomy and lymphadenectomy. Therefore, the cause of CTS may be related to the severity of the lymphedema rather than lymphadenectomy and the development of lymphedema in this case.

Selection of the proper surgical method to treat lymphedema may be important achieving a favorable result. Traditionally, LVA was considered for early-stage lymphedema but functional LVA may effectively reduce the volume of the limb and improve the subjective symptoms of patients with advanced-stage lymphedema [9]. LVA may benefit patients with advanced-stage upper extremity lymphedema when functional lymphatic vessels are identified. Recently, magnetic resonance lymphangiography and supermicrosurgery techniques have become available to successfully identify functioning lymphatic vessels and achieve favorable LVA results to improve lymphedema.

This study showed that the simultaneous release of the TCL and LVA could effectively reduce the volume and improve symptoms in the hand of a patient with advanced-stage lymphedema and CTS. Regarding the influence of lymphedema as a cause of neuropathy, previous studies have shown that lymphedema was a risk factor for aggravating CTS symptoms. Ganel et al. [13] were the first to identify an association between neuropathies and lymphedema in breast cancer patients who underwent mastectomy and lymphadenectomy. They showed that $62 \%$ of the patients diagnosed with CTS had lymphedema. Bozentka et al. [14] also showed that the symptom of hand numbness was related to the degree of lymphedema. In this study, we believe that lymphedema was not the cause of CTS, but that the symptoms of CTS could be worse according to the severity of lymphedema. The lymphedema of the patient in this study was characterized as International Society of Lymphology late stage 2 with severe dermal backflow in the entire extremities including the hand in ICG lymphography. However, the patient symptoms related to CTS were relieved when lymphedema symptoms such as heaviness, hardness, and largeness improved after complete congestive therapy. Further investigations are needed to clarify the association between CTS symptoms and the severity of lymphedema.

Lymphedema presents a difficult treatment problem because the pathophysiology of the condition involves multiple factors. Therefore, the selection of the proper surgical method for upper and lower extremity lymphedema may be important to achieving favorable results. For patients with stage 3 lymphedema, the clinical outcomes of the vascularized lymph node transfer for lymphedema may be superior to LVA. However, vascularized lymph node transfer is typically more invasive than LVA. In addition, donor site morbidity, long operation time, conspicuous scars, adhesion, and bulkiness following transfer at the wrist level are problems to overcome in vascularized lymph node transfer for lymphedema surgery. In contrast, LVA may be an alternative surgical option when functional lymphatic vessels can be identified using ICG lymphography. In addition, the time-cost necessary to complete LVA surgery was higher than that for vascularized lymph node transfer. An increase in operative time of one to two hours 
following the release of the TCL may improve the symptoms related to both CTS and lymphedema, consequently increasing the quality of life in CTS patients with advanced-stage lymphedema. Although a further case series with large numbers of patients and a long-term follow-up period is needed, simultaneous LVA and release of the TCL may be effective and safe in patients with advanced lymphedema and CTS.

\section{Conclusion}

This study was the first case report to demonstrate the effectiveness of simultaneous LVA and release of the TCL in a patient with advanced-stage lymphedema and concurrent CTS.

\section{Abbreviations}

CTS: Carpal tunnel syndrome; ICG: Indocyanine green; LVA: Lymphovenous anastomosis; TCL: Transverse carpal ligament

\section{Declarations}

\section{Ethics Approval and Consent to Participate}

The study protocol was approved by our Institutional Review Board of Korea University Guro Hospital and performed in accordance with the ethical standards of the Declaration of Helsinki.

\section{Consent for Publication}

Written informed consent was obtained from the patient.

\section{Availability of Data and Materials}

The material supporting the conclusion of the study is included in the article

\section{Competing Interest}

None of the authors have any conflict of interest to disclose.

\section{Funding}

Not applicable 


\section{Author's Contributions}

All authors were involved in preparation of this manuscript. SBK participated in the acquisition of clinical data and KCM wrote the manuscript. All authors have read and approved the final manuscript.

\section{Acknowledgements}

The author is indebted to David W. Chang, Deok-Woo Kim, Eul-Sik Yoon, and Eun-Sang Dhong who shared their experience in the microsurgical technique.

\section{References}

1. Schaverien MV, Coroneos CJ. Surgical Treatment of Lymphedema. Plast Reconstr Surg. 2019;144:738-58.

2. Forte AJ, Huayllani MT, Boczar D, Manrique OJ, Lu X, McLaughlin SA, et al. A Systematic Review of Peripheral Neuropathies in Breast Cancer-Related Lymphedema. Hand (N Y). 2020:1558944720963944.

3. Stubblefield MD, Kim A, Riedel ER, Ibanez K. Carpal tunnel syndrome in breast cancer survivors with upper extremity lymphedema. Muscle Nerve. 2015;51:864-9.

4. Vecht CJ. Arm pain in the patient with breast cancer. J Pain Symptom Manage. 1990;5:109-17.

5. Gunnoo N, Ebelin M, Arrault M, Vignes S. Impact of carpal tunnel syndrome surgery on women with breast cancer-related lymphedema. Breast Cancer Res Treat. 2015;152:683-6.

6. Koshima I, Nanba Y, Tsutsui T, Takahashi Y, Itoh S. Long-term follow-up after lymphaticovenular anastomosis for lymphedema in the leg. J Reconstr Microsurg. 2003;19:209-15.

7. Brorson H, Hoijer P. Standardised measurements used to order compression garments can be used to calculate arm volumes to evaluate lymphoedema treatment. J Plast Surg Hand Surg. 2012;46:410-5.

8. Pereira N, Lee YH, Suh Y, Choi DH, Suh HS, Jeon JY, et al. Cumulative Experience in Lymphovenous Anastomosis for Lymphedema Treatment: The Learning Curve Effect on the Overall Outcome. J Reconstr Microsurg. 2018;34:735-41.

9. Cha HG, Oh TM, Cho MJ, Pak CSJ, Suh HP, Jeon JY, et al. Changing the Paradigm: Lymphovenous Anastomosis in Advanced Stage Lower Extremity Lymphedema. Plast Reconstr Surg. 2021;147:199207.

10. DiSipio T, Rye $S$, Newman $B$, Hayes $S$. Incidence of unilateral arm lymphoedema after breast cancer: a systematic review and meta-analysis. Lancet Oncol. 2013;14:500-15.

11. Schou Bredal I, Smeby NA, Ottesen S, Warncke T, Schlichting E. Chronic pain in breast cancer survivors: comparison of psychosocial, surgical, and medical characteristics between survivors with and without pain. J Pain Symptom Manage. 2014;48:852-62. 
12. Langford DJ, Paul SM, West C, Abrams G, Elboim C, Levine JD, et al. Persistent arm pain is distinct from persistent breast pain following breast cancer surgery. J Pain. 2014;15:1238-47.

13. Ganel A, Engel J, Sela M, Brooks M. Nerve entrapments associated with postmastectomy lymphedema. Cancer. 1979;44:2254-9.

14. Bozentka DJBP CP, Schmidt S. Hand related disorders following axillary dissection for breast cancer. Univ PA Orthop J. 2001;14:35-7.

\section{Figures}

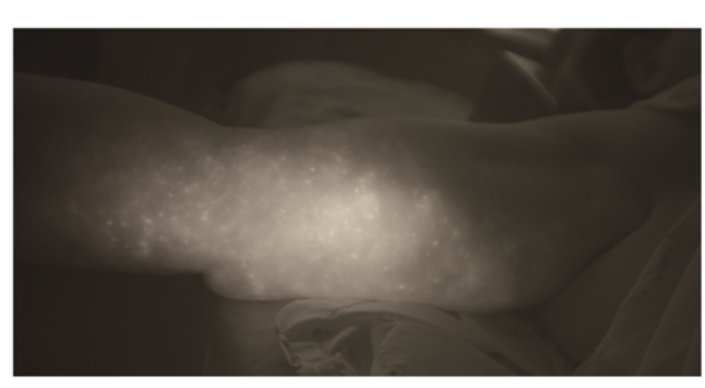

A

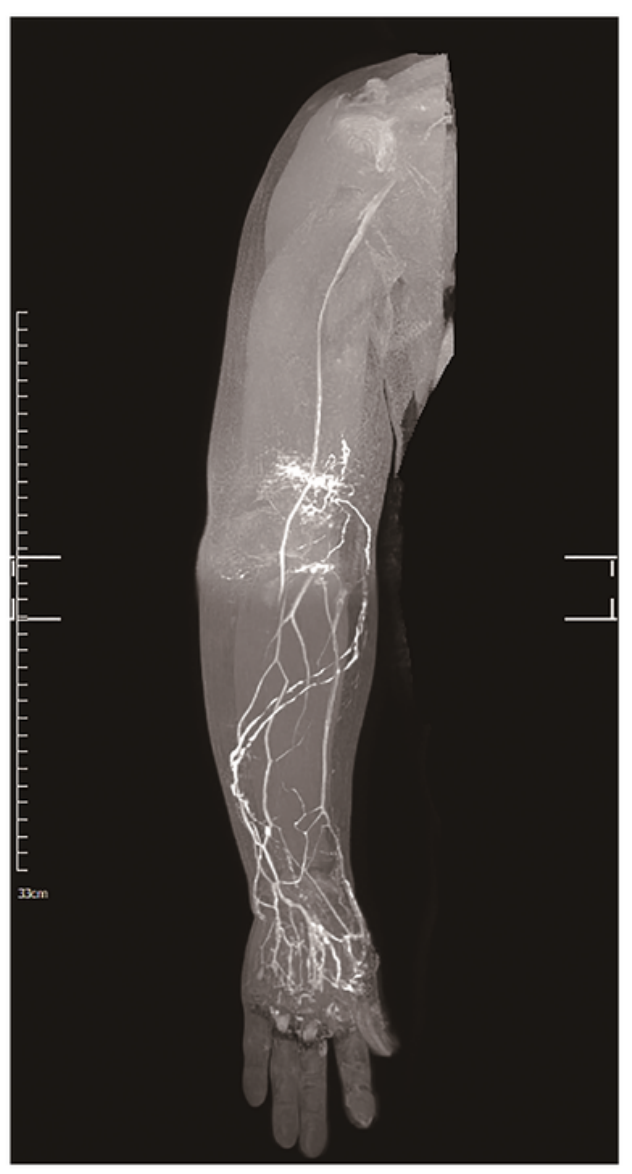

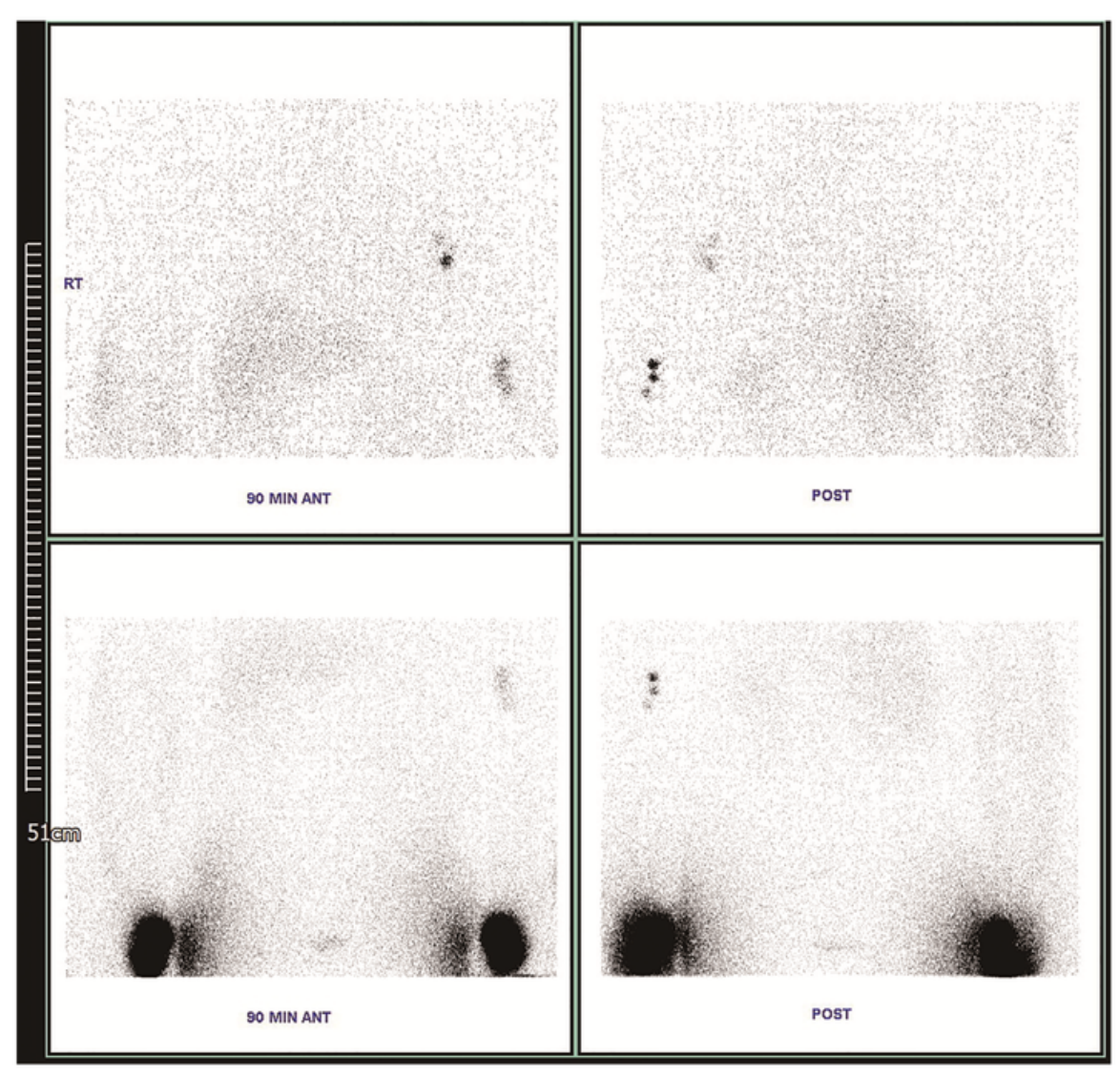

B 


\section{Figure 1}

A patient with right upper extremity lymphedema and carpal tunnel syndrome. (A) Indocyanine green lymphography. (B) Lymphoscintigraphy. (C) Magnetic resonance lymphangiography. This patient had International Society of Lymphology late stage 2 lymphedema with confluent dermal backflow and patent lymphatic vessels were visualized.

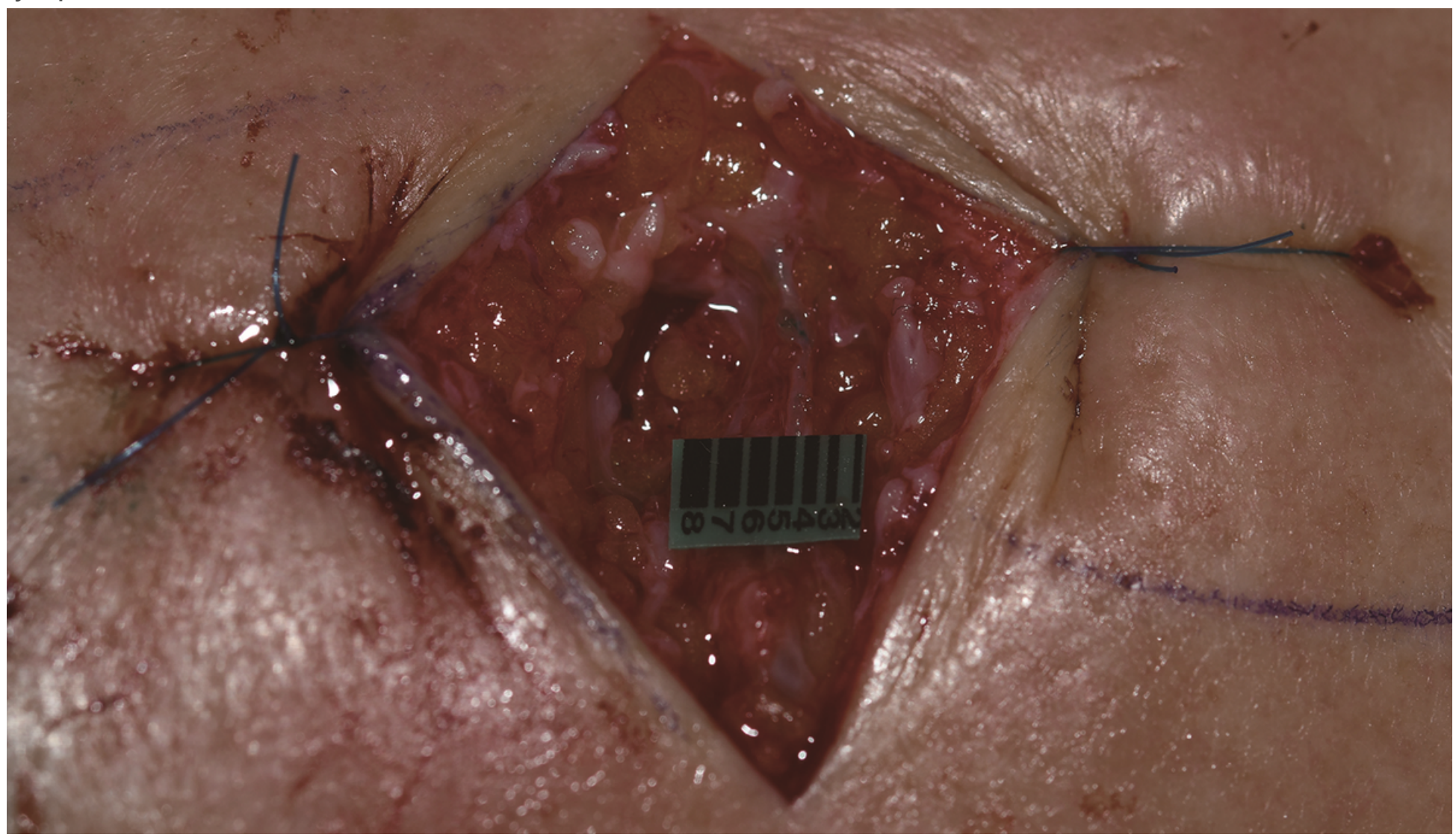

\section{Figure 2}

Lymphovenous anastomosis of the upper extremity. This patient underwent four lymphovenous anastomoses at the wrist, forearm, and anterocubital levels. An additional one hour of lymphovenous anastomosis may help patients relieve the symptoms of lymphedema and carpal tunnel syndrome.
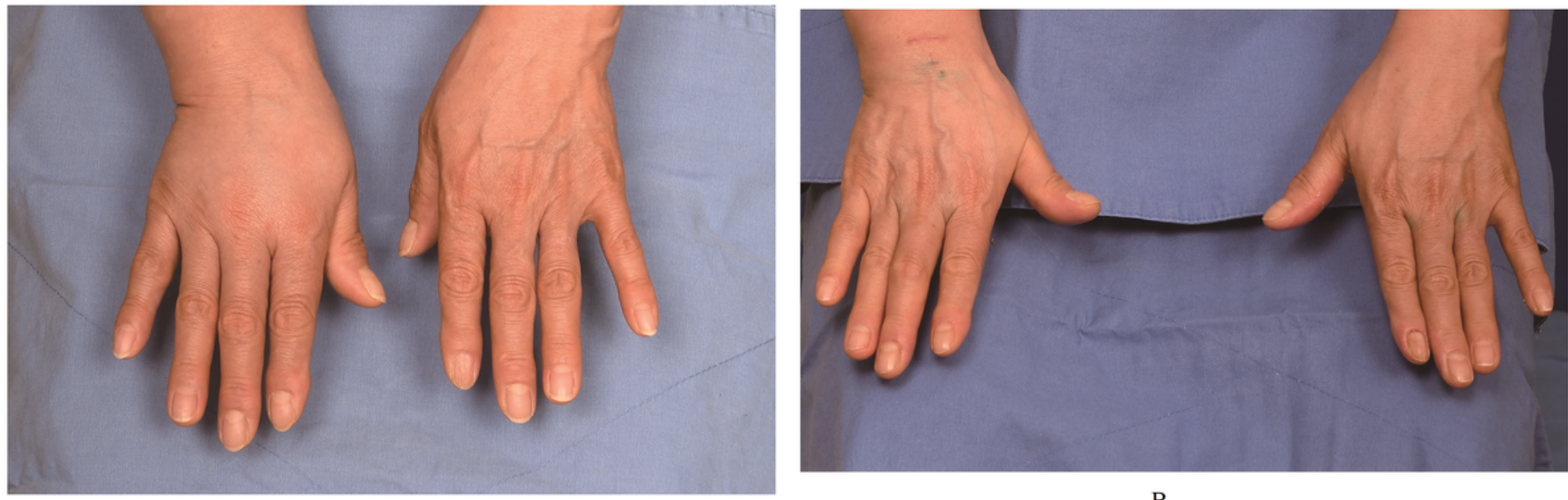


\section{Figure 3}

Preoperative and postoperative views of both hands. (A) Preoperative view. (B) Three-month postoperative view. This resulted in a significant reduction of the volume of the right hand and the patient reported improvement in the symptoms of both carpal tunnel syndrome and lymphedema. 